\title{
ESTIMATION OF INTER-CHANNEL PHASE SYNCHRONIZATION OF EEG SIGNALS IN THE RIDGES OF THEIR WAVELET SPECTROGRAMS IN PATIENTS WITH TRAUMATIC BRAIN INJURY
}

\section{Renata A. Tolmacheva, Yuri V. Obukhov}

Kotelnikov Institute of Radioengineering and Electronics of RAS, http:/ /www.cplire.ru/ 11/7, Mokhovaya str., Moscow 125009, Russian Federation

\section{Lyudmila A. Zhavoronkova}

Institute of Higher Nervous Activity and Neurophysiology of RAS, http://www.ihna.ru/ 5a, Butlerova str., Moscow 117485, Russian Federation tolmatcheva@ya.ru,yuvobukhov@mail.ru, lzhavoronkova@hotmail.com

Abstract. Identical inter-channel phase coherency of electroencephalogram (EEG) signals is determined for healthy subjects during cognitive and motor tests. EEG signal phase is evaluated at the points of it wavelet-spectrogram ridge. Areas of interest of the cortex at cognitive and motor tests for group of healthy subjects are determined. Inter-channel EEG phase coherency for patients after a traumatic brain injury are represented.

Keywords: electroencephalogram, wavelet-spectrogram, ridge, phase synchronization

UDC 53.088

Bibliography - 11 references

Received 18.06.2019, accepted 27.06.2019

RENSIT, 2019, 11(2):243-248

DOI: $10.17725 /$ rensit.2019.11.243

\section{Contents}

1. INTRODUCTION (243)

2. Methods (244)

3. Results (245)

4. Conclusion (247)

REFERENCES (247)

\section{INTRODUCTION}

Usually coherency or phase connectivity of EEG signals use to the estimation of inter-channel EEG connectivity [1-3]. The coherency $\operatorname{Coh}_{\mathrm{xy}}(f)$ is determined by the normalized complex cross correlation of signals $x$ and $y$ :

$$
\begin{aligned}
C_{x y}(f) & =\frac{S_{x y}(f)}{\left(S_{x x}(f) S_{y y}(f)\right)^{1 / 2}}, \\
C_{0} h_{\mathrm{xy}}(f) & =\left|C_{\mathrm{xy}}(f)\right| .
\end{aligned}
$$

In coherent analysis, due to the modulus of normalized complex cross correlation $C_{\mathrm{xy}}(f)=1$, the coherency $\operatorname{Coh}_{\mathrm{xy}}(f)$ is averaged in different of time intervals and in a certain frequency interval that is determined using neurophysiological data. Normally, such intervals correspond to the delta $(2-4 \mathrm{~Hz})$, theta (4-8 Hz), and alpha (8-12 Hz) EEG rhythms. Such an averages and the presence of the threshold level of coherence that is used to select the phase-coupled pairs of signals are disadvantages of the coherent analysis that lead to instability in the determination of the inter-channel phase synchronization of EEG signals. These disadvantages are considered in detail in [4]. The validity of the coherent analysis of essentially non-stationary EEG signals is questioned $[2,4]$.

Analytical signal also can be used for the estimation of phase connectivity $x^{*}(t)=x(t)+$ $i H(x(t))$, where $H(x(t))$ is the Hilbert transform. The phase synchronization of two signals takes place when [5]:

$\left|\Phi_{\mathrm{x}, \mathrm{y}}(t)\right| \leq$ const

where $\Phi_{\mathrm{x}, \mathrm{y}}(t)=n \Phi_{\mathrm{x}}(t)-m \Phi_{\mathrm{y}}(t), \Phi$ is the phase of the signal, $n, m$ are integers.

We proposed an approach to the estimation of the inter-channel phase connectivity of the EEG [6] based on the phases calculation and comparison of the signals at the points of the ridges of their wavelet spectrograms having the property of stationarity of the phase, which does not exist in both of the above approaches. Under this the approach, phase-coupled pairs of EEG signals are considered for the group of healthy subjects and for the group of patients with traumatic brain injury during two cognitive and motor tests. 


\section{METHODS}

The ridges of the wavelet spectrograms are defined as the points of the stationary phase [7] that is in them the derivative of the phase with respect to time is equal to the frequency. In [8], devoted to computer modeling of smooth music, it was shown that at the points of time- and frequency-asymptotic ridges of wavelet spectrograms the phase is stationary in the sense that at these points $d \Phi / d t \approx \omega$.

The EEG signal responds the asymptotic properties under the conditions:

$$
\left|\frac{d \Phi(t)}{d t}\right|>>\left|\frac{1}{A(t)} \frac{d A(t)}{d t}\right|,\left|\frac{1}{A(t)} \frac{d A(t)}{d t}\right| \ll<\left|\frac{1}{\psi(t)} \frac{d \mid \psi(t)}{d t}\right| .
$$

The approach to estimating the inter-channel phase connectivity of the EEG at the points of the ridges of their wavelet-spectrograms with the stationary phase is considered as an inverse task to the problem of modeling ridges. In [6] it is shown that for the amplitude and phase of the amplitudemodulated signal $x(t)=A(t) e^{i \Phi(t)}$ is true:

$$
A(t) \approx\left|W\left(t, f_{r}\right)\right| \text { and } \Phi(t) \approx \arctan \left(\frac{\operatorname{Im} W\left(t, f_{r}\right)}{\operatorname{Re} W\left(t, f_{r}\right)}\right) .
$$

Based on this, first we find the ridge with the maximum value $\left|W\left(t, f_{\mathrm{r}}\right)\right|$ at each reference point $\tau_{\mathrm{i}}$ of the Morlet wavelet:

$$
\begin{aligned}
& W(\tau, f)=\sqrt{f} \int x(t) \psi((t-\tau) f) d t, \\
& \psi(\eta)=\frac{1}{\sqrt{\pi F_{b}}} e^{2 \pi i F_{c} \eta} e^{-\frac{\eta^{2}}{F_{b}},}
\end{aligned}
$$

where $F_{\mathrm{b}}=F_{\mathrm{c}}=1$.

Then, we calculate the phase difference of two signals $x(t)$ and $y(t)$ at the points of the ridges $t_{\mathrm{k}}$ of their wavelet spectra and select const to estimate their phase synchronization.

In this case, the points of wavelet spectrograms with nonstationary phase are not included in the estimation of the phase of signals. However, such points are used in the estimation of coherence and phase with the aid of the Hilbert transform and coherency approach [1-3]. When the ridge points do not satisfy the condition for asymptotic ridges (4), we obtain errors in the calculations of the phase difference. However, we assume that such an error is significantly less than the errors related to the averaging of the phase difference in a certain interval of the ridge frequencies. After elimination the frequency range of the processed ridge, we employ the above algorithm to select the next ridge with a lower power spectral density in a different frequency range.

The international system of the location of scalp electrodes «10-20\%» is used to the EEG record (Fig. 1). The records of 19-channel EEG were analyzed, therefore the number of pairs of channels is 171 for the group of the healthy subjects (8 people) and for the group of the patients with traumatic brain injury (5 people).

The sampling frequency of the EEG was 250 $\mathrm{Hz}$ in the processing of EEG signals. The original signals were recorded with a high-pass filter with cut-off frequency of $0.5 \mathrm{~Hz}$, a low pass filter with a cut-off frequency of $70 \mathrm{~Hz}$. Then, a $50 \mathrm{~Hz}$ notch filter and a Butterworth filter were used. The signals were filtered by a fourth-order Butterworth bandpass filter with a bandwidth of 2 to $10 \mathrm{~Hz}$. The duration of every EEG record was 60 seconds. Also, the removing of outliers in the EEG signals was with the Huber's X84 method [9].

Fig. 2 shows an example of the power spectral density of Morlet wavelet spectrograms in a pair of EEG channels with selected points of the maximum spectral power density (ridge). Fig. 3 shows the phases time dependencies of two EEG signals $x(t)$ and $y(t)$ at the points of the ridges of their wavelet spectrograms.

Then, we plot the histograms of values of the phase difference of two signals $x(t)$ and $y(t)$ as in [5].

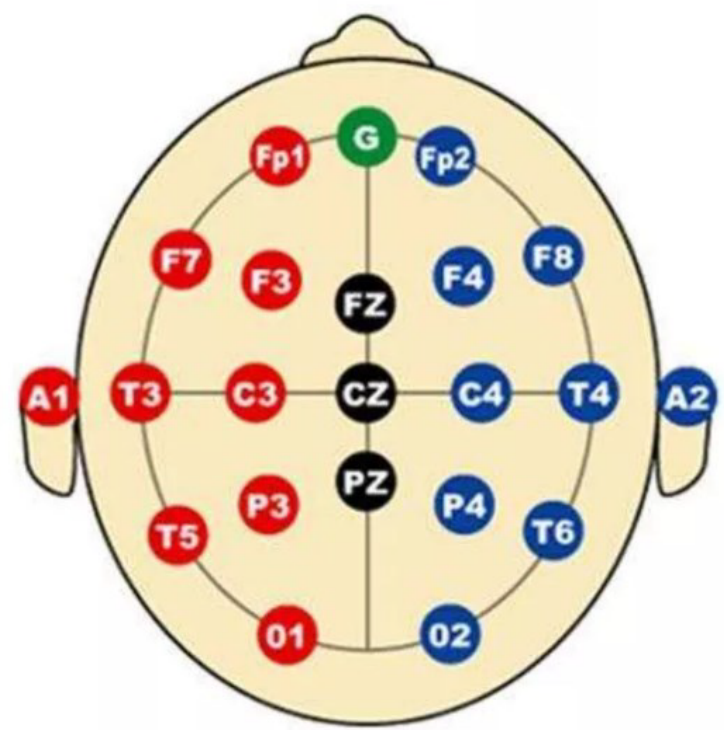

Fig. 1. The international system of the location of scalp electrodes $\langle 10-20 \%\rangle$. 

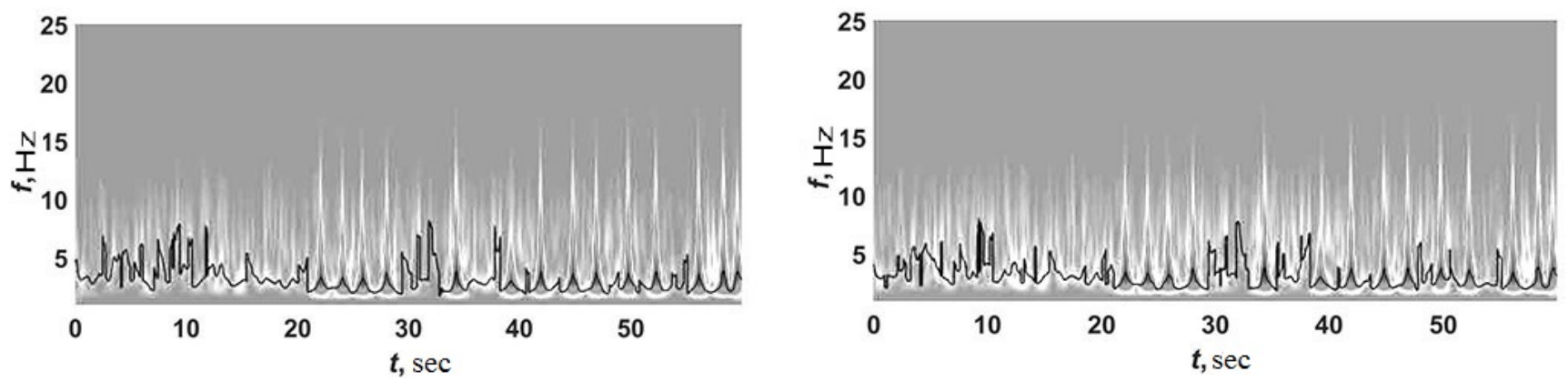

Fig. 2. Morlet wavelet spectrograms of the signal for a pair of the EEG channels with the selected ridge: a) first channel; b) second channel.

Fig. 4 represents the normalized histograms $\rho_{x, y}$ $=n_{\mathrm{x}, \mathrm{y}} / \mathrm{N}$, where $n_{\mathrm{x}, \mathrm{y}}$ is number of reference points of ridges with the discretization of the histogram $\left|\Delta \Phi_{\mathrm{x}, \mathrm{V}}\left(t_{\mathrm{k}}\right)\right|<0.05 \pi, N$ is a total number of EEG signal reference points during the test. The first pair of channels Fp1-Fp2 (Fig. 4a) can be referred to a phase-coupled pair. Another pair Fp1-O2 (Fig. 4b) can be referred to a phase-unconnected pair. Fig. 4a shows that $\rho_{x, y}<0.1$ can be considered as a background. We consider the threshold $\rho_{x, y}^{t h r}=0.1$ and we will assume that the values of the portions of the ridge points above it will correspond to the phase-coupled pairs of channels. Note that the phase difference of two EEG channels (e.g., Fp1Fp2) can be used to characterize the phase-locking dynamics. Fig. 5 presents the plot of the phase difference for two channels of the EEG signal (Fp1-Fp2) vs. time.

Distributions graphs of the maximum values of portions $\max \rho_{x, y}$ over pairs of EEG channels, based on the histograms obtained (Fig. 4), were arranged in the ascending order $\max \rho_{x, y}$ without tests, with the cognitive calculation-logical test, with the cognitive spatial-pattern test and with the motor test are represented in Fig. $\mathbf{6}$.

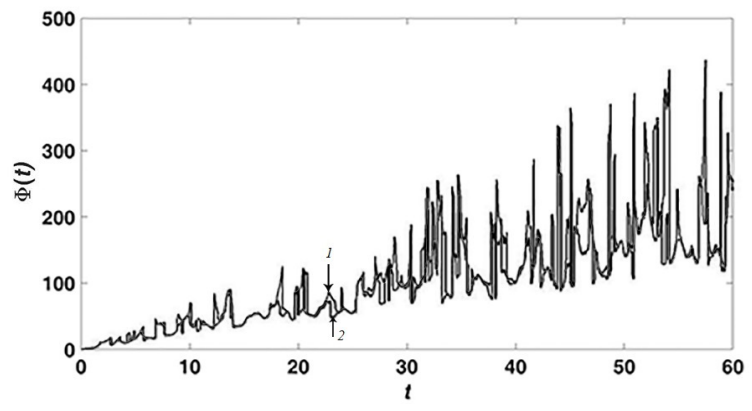

Fig. 3. Dependences of phases on time of two EEG channels. Line 1 is the phase of EEG signal of FP1channel. 2 - the phase of EEG signal of FP2 channel.

\section{RESULTS}

By the new approach of estimation [10], the interchannel EEG the phase synchronization during two cognitive (calculation-logical and spatial-pattern) and motor tests for the group healthy subjects (8 people) and for the group patients with traumatic brain injury are considered. During the cognitive calculation-logical test, doctor randomly called some items that belong to the category "clothes" or "food" to the subject. During the test, the subject counts in the mind the quantity of items belonging to one of these categories. At the end of the test, he declares the result of quantity of items. When performing the cognitive spatial-pattern test, the doctor randomly called the time. The subject must imagine in the mind the dial of the clock and the position of the clock hands on it in accordance with the time mentioned. If both clock hands are in the same half of the dial, he says "yes," and if they are in different halves, he keeps silent. When performing the motor test, the subject stands on a stabilographic platform. The position of his center of gravity is displayed on the screen. He must hold center of gravity inside a circle of a certain diameter. The duration of every test was 60 seconds. The EEG record was performed both during the tests and without them.

From the dependences of Fig. 6 it can be distinguished phase-coupled pairs of the EEG
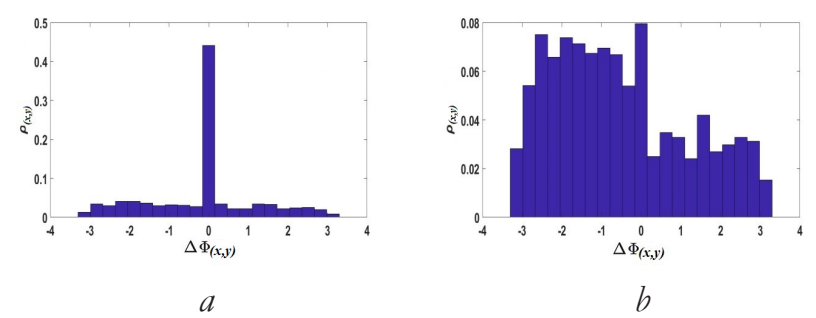

Fig. 4. Histograms of portions $\rho_{x, y}$ for the phase difference at ridge points of the wavelet spectrograms for two EEG channels: a) phase-coupled pair of EEG channels; b) uncoupled pair of EEG channels. 


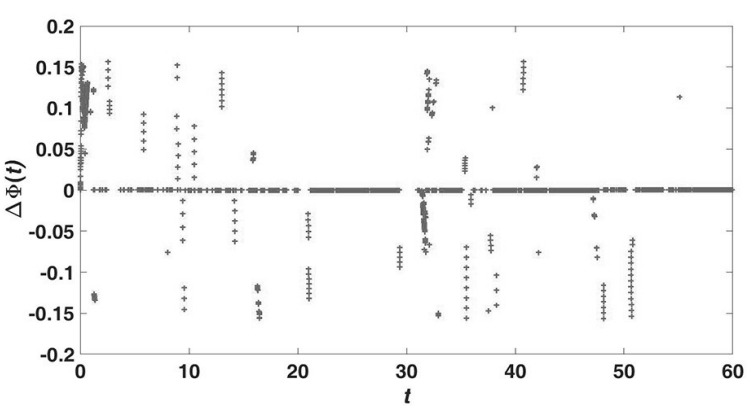

Fig. 5. The phase difference of two EEG signals (Fp1-Fp2) vs. a time.

channels that appear only during the test, by comparing the phase-coupled pairs of the EEG record without the test and at the time of the EEG records during one of the tests. Phase-coupled pairs that are absent in EEG records without tests and that appear only during tests are shown in Fig. 7 for the group of the healthy subjects and in Fig. 8 for the group of patients with traumatic brain injury.

Based on the obtained pairs of EEG channels (Fig. 6), it is possible to find phase-coupled pairs of the EEG channels that appear in a group of 8 healthy subjects during the cognitive calculationlogical test, the cognitive spatial-pattern test and motor test (Fig. 7).

Fig. 7 shows that the greater number of the phasecoupled pairs of the EEG channels that appear in the group of the healthy subjects during the cognitive calculation-logical test is in the left hemisphere. And, the greater number of the phase-coupled pairs of the EEG channels that appear in the group of the healthy subjects during the cognitive spatial-pattern test is in the right hemisphere. In accordance with published works, the prefrontal regions of the left

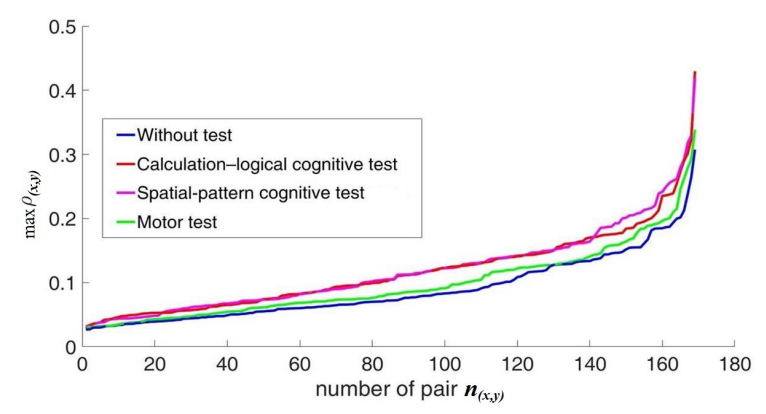

Fig. 6. Distributions graphs of the maximum values of portions $\max \rho_{x, y}$ over pairs of EEG channels arranged in the ascending order max $\rho_{x, y}$. Numbers of pairs EEG channels are plotted on the abscissa. Purple line is the EEG record without test; red line is the EEG record with the calculation-logical cognitive test; blue line is the EEG record with the spatial-pattern cognitive test; green line is the EEG record with the motor test.

and right hemispheres are predominantly activated in the calculation-logical and spatial-pattern tests, respectively for healthy subjects [11]. Our method confirms this.

Also, a larger number of phase-coupled pairs reflects the different efforts required to perform each of the tests, which, according to the complexity of performing from easy to difficult, can be arranged in the following sequence: motor test - calculationlogical cognitive test - spatial-pattern cognitive test.

Fig. 8 shows phase-coupled pairs of channels for the group of patients with traumatic brain injury (5 people). Phase-coupled pairs of EEG channels for each patient are represented by special color.

Fig. 8 represents that phase-coupled pairs that appear in the group of patients with traumatic brain injury practically are absent. Coincident phasecoupled pairs are represented in only two patients during the cognitive calculation-logical test: Fp1 - Fp2

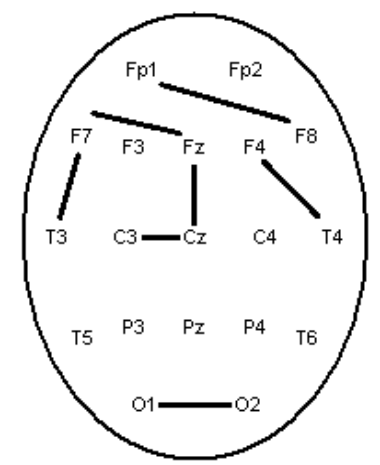

a

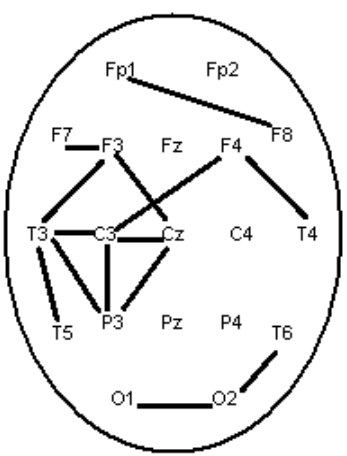

$b$

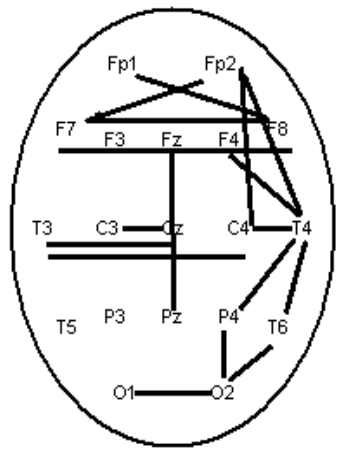

c

Fig. 7. Phase-coupled pairs that appear in the group of 8 bealthy subjects during calculation-logical cognitive test, spatial-pattern cognitive test and motor test. a) motor test; b) calculation-logical cognitive test; c) spatial-pattern cognitive test. 

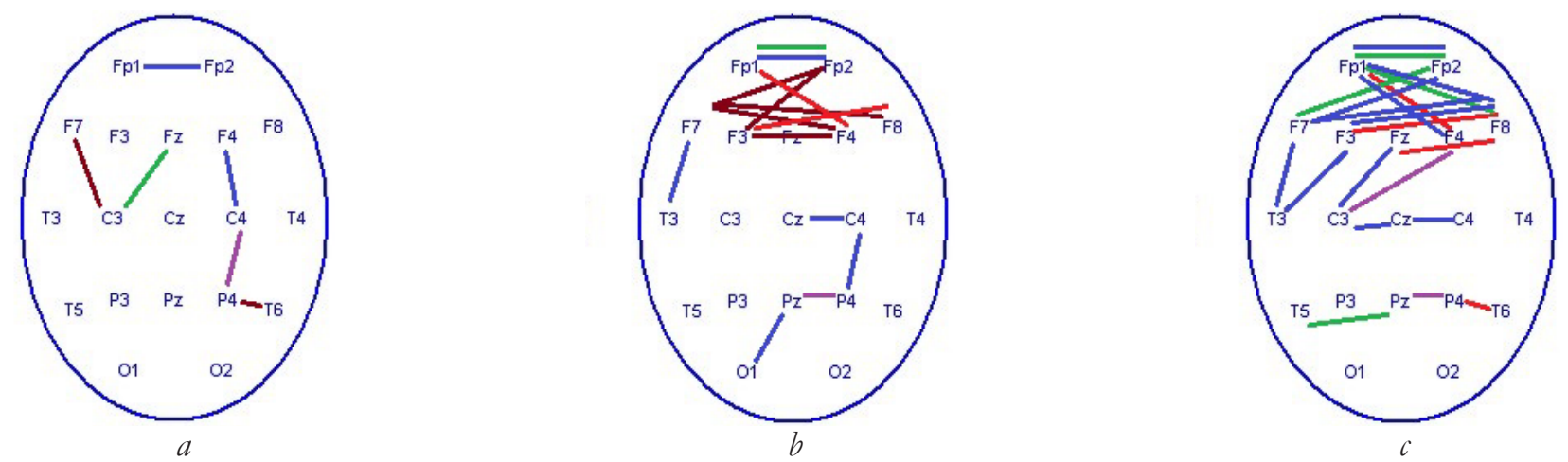

Fig. 8. Phase-coupled pairs that appear in the group 5 patients with traumatic brain injury during calculation-logical cognitive test, spatial-pattern cognitive test and motor test. a) motor test; b) calculation-logical cognitive test; c) spatial-pattern cognitive test. Phasecoupled pairs of EEG channels for each patient are represented by special color.

and coincident phase-coupled pairs are represented in three patients during the cognitive spatial-pattern test: Fp1-Fp2, Fp1-F8, Fp2-F7, F3- F8 и Fp1-F4. These are EEG channels of the frontal region of the brain. The activation of the left hemisphere during the performance of the cognitive calculation-logical test is absent by patients with traumatic brain injury. Also, the activation of the right hemisphere during the performance of the cognitive spatial-pattern test is absent by patients with traumatic brain injury.

\section{CONCLUSIONS}

By the new approach of the estimation the interchannel phase synchronization of the EEG signals in different channels, based on the calculation and comparison of the phases of the signals at the points of the wavelet spectrograms ridges, phasecoupled pairs of EEG channels that appear for the group of 8 healthy subjects during two cognitive and motor tests are considered. Areas of interest of the cortex at two cognitive (calculation-logical and spatial-pattern) and motor tests for healthy subjects are determined. The prefrontal regions of the left hemisphere are predominantly activated in the calculation-logical cognitive test for healthy subjects, but these regions aren't activated for patients with traumatic brain injury. The prefrontal regions of the right hemisphere are predominantly activated in the spatial-pattern cognitive test for healthy subjects, but these regions aren't activated for patients with traumatic brain injury. The definition of phase-coupled pairs of EEG signals can be useful for the monitoring the treatment of people with traumatic brain injury. The recovery of phase-coupled EEG pairs can be used as indicator of proper treatment.

\section{ACKNOWLEDGMENTS}

The work was carried out within the framework of the state task and partially was supported by the Russian Foundation for Basic Research, the project no. 18-07-00609.

\section{REFERENCES}

1. Wending F, Ansari-Asl K, Bartolomei F, Senhadji L. From EEG signals to brain connectivity: a model based evaluation of interdependence measures. Journal of Neuroscience Methods, 2009, 183(1):9-18.

2. Nolte G, Bai O, Wheaton L, Mari Z, Vorbach S, Hallett M. Identifying true brain interaction from EEG data using the imaginary part of coherency. Clin. Neurophysiol., 2004, 115:2292-2307.

3. Zhan Y, Halliday D, Jiang P, Liu X, Feng J. Detecting time-dependent coherence between non stationary electrophysiological signals. A combined statistical and time-frequency approach. Journal of Neuroscience Methods, 2006, 156:322-332.

4. Kulaichev A. Ob informativnosti kogerentnogo analiza [On the informativeness of coherent analysis.]. Zh. Vysshei Nerv. Deyatel'nosti im. I. P. Pavlova, 2009, 59(6):766-775 (in Rbss.).

5. Tass P, Rosenblum MG, Weule J, Kurths J, Pikovsky A, Volkmann J, Schnitzler A, Freund H-J. Detection of n:m Phase Locking from Noisy Data: Application to Magnetoencephalography. Physical Review Letters, 1998, 81(15):3291-3294.

6. Tolmacheva RA, Obukhov YuV, Polupanov AF, Zhavoronkova LA. New Approach to Estimation of Interchannel Phase Coupling of Electroencephalograms. Journal of Communications Technology and Electronics, 2018, 63(9):1070-1075.

7. Mallat SG. $A$ Wavelet Tour of Signal Processing. The Sparse Way. Burlington, Academic Press, 2009, 
$562 \mathrm{p}$.

8. Guilleemain P, Kronland-martinet R. Characterization of Acoustic Signals Through Continuous Linear Time-Frequency Representations. Proc. IEEE, 1996, 84(4):561585.

9. Hampel FR, Ronchetti EM, Rousseeuw PJ, Stahel WA. Robust Statistics: The Approach Based on Influence Functions. Wiley, 1986, 526 p.

10. Obukhov YuV, Tolmacheva RA, Polupanov AF, Zhavoronkova LA. On an approach to assessing the inter-channel phase synchronization of electroencephalogram signals. Proc. Intern. Conf. Information Technology and Nanotechnology (ITNT2018, Samara, Russia, April 24-27, 2018). Samara, CEUR, 2018, pp. 84-88; http://ceur ws.org/Vol 2212/paper11.pdf.

11. Zhavoronkova L, Shevtsova T, and Maksakova O. How Does Human Brain Simultaneously Solve Two Problems? LAP LAMBERT, Academic Publ., Saarbrücken, 2017. 